\title{
Oral polymorphonuclear neutrophil characteristics in relation to oral health: a cross-sectional, observational clinical study
}

\author{
Patrick Rijkschroeff ${ }^{1}$, Ineke DC Jansen ${ }^{1}$, Fridus A van der Weijden ${ }^{1}$, Bart JF Keijser ${ }^{2,3,4}$, Bruno G Loos ${ }^{1}$ \\ and Elena A Nicu ${ }^{1}$
}

Polymorphonuclear neutrophils (PMNs) have a major role in the innate immune system. However, little is known about PMN contribution in relation to oral health. The objective of this study was to investigate the numbers and functional characteristics of oral PMNs (oPMNs) compared with circulatory PMNs (cPMNs). Oral rinse and venous blood samples were obtained from 268 systemically and orally healthy volunteers in a cross-sectional observational study. PMN counts, cell cycle analysis and cellular activation state were investigated. Also, reactive oxygen species (ROS) production was analyzed, with and without bacterial stimulation (Fusobacterium nucleatum). In males, $1.2 \times 10^{6} \pm 1.0 \times 10^{6}$ oPMNs were collected, and showed a tendency to correlate with the levels of gingival bleeding $(r=0.215, P=0.008)$. Comparable oPMNs counts were found among females $\left(1.0 \times 10^{6} \pm 0.7 \times 10^{6}\right)$. More late-stage apoptotic/necrotic cells were found among the oPMNs $(53.1 \%)$ compared with the cPMNs $(8.5 \% ; P<0.001)$. Without additional stimulation, oPMNs were more activated than cPMNs, as indicated by higher expression of CD11b, CD63 and CD66b, and higher constitutive ROS levels $(P<0.001)$. Notably, in response to bacterial stimulation, oPMNs released comparable ROS levels as cPMNs $(P=0.042)$. In conclusion, this study provides data on viable oPMNs showing high levels of activation in orally and systemically healthy individuals, free of apparent caries lesions and periodontal disease. These data suggests that although the oPMNs are in a more mature stage of their life cycle compared with the cPMNs, oPMNs are still responsive to stimulation, which indicates their functional potential and possible contribution to a healthy oral ecosystem. International Journal of Oral Science (2016) 8, 191-198; doi:10.1038/ijos.2016.23; published online 12 August 2016

Keywords: apoptosis; degranulation; neutrophils; oral health; reactive oxygen species

\section{INTRODUCTION}

The oral ecosystem is characterized by the interplay between the oral microbiota, salivary biochemistry, and host immune responses. Although the oral cavity harbors billions of microorganisms, including over 1000 different identified species, ${ }^{1-2}$ overt infections rarely occur and soft tissue wounds in the mouth heal remarkably well. ${ }^{3}$

Functional polymorphonuclear neutrophils (PMNs) are of paramount importance in innate immunological processes, including the maintenance of oral health. ${ }^{4-5}$ PMNs are terminally differentiated leukocytes, which are able to respond quickly to host and bacterially derived chemotactic factors. ${ }^{6}$ They perform several protective functions, such as phagocytosis with the generation of reactive oxygen species (ROS). ${ }^{7-8}$ In addition, during PMN activation, degranulation occurs, and products from the intracellular pools of granules, such as antimicrobial peptides, myeloperoxidase, proteolytic enzymes and lactoferrin, are released. ${ }^{6}$ Under normal circumstances, these mechanisms contribute to the neutralization and elimination of microorganisms and damaged cells. ${ }^{9-10}$
The oral cavity and the gastrointestinal tract are both heavily colonized sites. In the gastrointestinal tract, the local equilibrium involves functional PMNs, which inactivate luminal microbes that translocate across the epithelium and invade deeper into the local mucosa. ${ }^{6}$ Oral PMNs (oPMNs) may have a similar surveillance function as that described for intestinal PMNs.

Most of the current knowledge regarding PMN functionality in the oral cavity is derived from studying oral diseases ${ }^{11-18}$ and little is known about oral health. Only a few small-scale studies have addressed oPMNs in non-diseased individuals. ${ }^{19-21}$

Notably, oral health is often confused with the absence of disease. A healthy oral ecosystem maintains homeostasis by neutralizing stressing agents and repairing possible damage. ${ }^{22}$ Recent studies showed that PMN gene expression can change during inflammatory responses and that different PMN phenotypes may be found within different environments. $^{21,23}$ Understanding the phenotypic changes of the oPMNs that occur after migration from the circulatory system to the

\footnotetext{
${ }^{1}$ Department of Periodontology, Academic Centre for Dentistry Amsterdam, Amsterdam, The Netherlands; ${ }^{2}$ Department of Preventive Dentistry, Academic Centre for Dentistry Amsterdam, Amsterdam, The Netherlands; ${ }^{3}$ Microbiology and Systems Biology, TNO Earth, Environmental and Life Sciences, Zeist, The Netherlands and ${ }^{4}$ Top Institute Food and Nutrition, Wageningen, The Netherlands

Correspondence: Dr EA Nicu, Department of Periodontology, Academic Centre for Dentistry Amsterdam (ACTA), Gustav Mahlerlaan 3004, 1081 LA Amsterdam, The Netherlands E-mail: e.nicu@acta.nl

Accepted 3 May 2016
} 
oral cavity, might give new insights into how these innate immune cells function in a physiologic state.

Describing the oPMNs will help to define the characteristics of the healthy oral ecosystem and will establish reference values for future research. We aimed to analyze the numbers, the cell cycle distribution, cell activation status, as well as stimulated- and unstimulated ROS production of the PMNs from the oral cavities of non-smoking, orally and systemically healthy volunteers. These characteristics were compared to those of circulatory PMNs (cPMNs) and evaluated for possible differences relative to donor gender. We hypothesize that the oPMNs have a crucial role in the maintenance of a healthy oral cavity and therefore do not differ from the cPMNs regarding functional capabilities.

\section{MATERIALS AND METHODS}

\section{Study population}

The study was carried out within the framework of the Top Institute Food and Nutrition as part of the project "Estimating the boundaries for a healthy oral ecosystem in young individuals" (Netherlands Trial Register number NTR3649), in a cross-sectional, single-center, observational clinical study at the Academic Center for Dentistry Amsterdam, The Netherlands. Volunteers were invited for screening (October 2012 until March 2013) if they had visited their general dentist within the previous year and diagnosed to be without oral or dental problems. The recruitment protocol, inclusion and exclusion criteria are described in previous studies. ${ }^{24-25}$ Notably, exclusion criteria were: smoking, presence of systemic disease, overt dental caries, interproximal restorations between the first and second, or the second and third upper molars, periodontitis or other oral infections, recent use of antibiotics, and use of anti-inflammatory drugs or other prescribed medication (except for oral contraceptives) which could interfere with the outcome of this study. Participants were instructed not to eat, drink, chew gum, or perform strenuous physical exercise before the appointment, and not to brush their teeth the evening before or on the morning of the appointment.

For this current study and the previous investigations on "Estimating the boundaries for a healthy oral ecosystem in young individuals", ${ }^{24-25}$ the number of included individuals was based on preliminary analyses in a similar study population, investigating the scores of bleeding upon marginal probing (BOMP). ${ }^{26}$ These showed a bell curved distribution and by enrolling at least 250 subjects, we expected the extremes (defined by BOMP scores of $\leq 40$ and $\geq 70 \%$ ) to be populated by $30-40$ individuals.

\section{Ethical considerations}

All participants were informed about the purpose of this study, received written information, and gave signed consent before the start of this study in accordance with the Declaration of Helsinki (2008) of the World Medical Association and approximating Good Clinical Practice guidelines. The study protocol was reviewed and approved by the Medical Ethics Review Committee of the Academic Medical Centre of Amsterdam (2012_210\#B2012406). The trial was monitored during its course, as assigned by the commissioning party (ACTA Dental Research BV), to ensure compliance with the protocol, regulations and guidelines, adequacy of the equipment and facilities and satisfactory data collection.

\section{Clinical collection procedures for the oPMNs and cPMNs}

The collection and isolation protocol for the oPMNs was based on previously described methods. ${ }^{19,27}$ The oral rinse samples were always taken in the morning (08:30-10:30 hours). The volunteers were asked to rinse the oral cavity thoroughly with $20 \mathrm{~mL}$ of phosphate-buffered saline solution (PBS; Gibco, Paisley, UK), containing $0.1 \%$ gelatin for $30 \mathrm{~s}$ and to expectorate into two $50 \mathrm{~mL}$ centrifuge tubes (SigmaAldrich Chemie B.V., Zwijndrecht, The Netherlands). This procedure was repeated three times, each after a 4.5 -min intermission, and the rinse samples were kept on ice until the end of the collection process.

Venous blood samples were drawn from the antecubital fossa into a $6 \mathrm{~mL}$ sodium heparin blood collection tube (BD Vacutainer, Breda, The Netherlands) and maintained at room temperature for a maximum of $2 \mathrm{~h}$, until the $\mathrm{CPMN}$ isolation procedure.

\section{Dental plaque and gingival bleeding assessment}

After clinical sampling, plaque levels were scored. Six surfaces on each tooth were assessed as described by Van der Weijden et al. ${ }^{28}$ The level of gingival inflammation was assessed according to the BOMP index as described by Lie et al. ${ }^{29}$ Mean plaque percentages and mean BOMP percentages were calculated at a subject level. The complete protocol and the results of plaque and bleeding assessments are described in a previous study. ${ }^{24}$

\section{oPMNs isolation procedure}

The tubes containing rinse samples were vortexed for $1 \mathrm{~min}$ and centrifuged using an Eppendorf AG 5804R centrifuge (Eppendorf, Hamburg, Germany) at 500 relative centrifugal force (RCF) for $10 \mathrm{~min}$ at $4{ }^{\circ} \mathrm{C}$. The supernatant was discarded, and the pellet was resuspended in $2 \mathrm{~mL}$ PBS and passed through $31.5 \mu \mathrm{m}$ and $10.0 \mu \mathrm{m}$ nylon meshes (Nedfilter, Almere, The Netherlands). The filtrated fraction was centrifuged (500 RCF, $10 \mathrm{~min}, 4^{\circ} \mathrm{C}$ ), and the remaining pellet was resuspended in supplemented PBS (Gibco, Paisley, UK) with $1.5 \mathrm{mmol} \cdot \mathrm{L}^{-1} \mathrm{CaCl}_{2}, 1 \mathrm{mmol} \cdot \mathrm{L}^{-1} \mathrm{MgCl}_{2}-\mathrm{H}_{2} \mathrm{O}$ (sPBS).

\section{cPMNs isolation procedure}

The cPMNs were isolated using a density-gradient centrifugation method. The blood samples were 1:1 diluted with PBS-containing $1 \%$ trisodium citrate, $\mathrm{pH} 7.4$ and layered on top of $15 \mathrm{~mL}$ Lymphoprep (Axis-Shield, Oslo, Norway). The tubes were centrifuged at 800 RCF for $30 \mathrm{~min}$ at $20^{\circ} \mathrm{C}$. The red cell layer, containing granulocytes and erythrocytes, was harvested and subsequently the erythrocytes were lysed in $\mathrm{NH}_{4} \mathrm{Cl}$ solution $\left(155 \mathrm{mmol} \cdot \mathrm{L}^{-1} \mathrm{NH}_{4} \mathrm{Cl}, 10 \mathrm{mmol} \cdot \mathrm{L}^{-1}\right.$ $\mathrm{KHCO}_{3}, 0.1 \mathrm{mmol} \cdot \mathrm{L}^{-1}$ EDTA at $\mathrm{pH} 7.4$ ). After $15 \mathrm{~min}$ incubation on ice, samples were centrifuged at $400 \mathrm{RCF}$ for $5 \mathrm{~min}$ at $4{ }^{\circ} \mathrm{C}$. Subsequently, the erythrocytes lysis step was repeated before the samples were washed with PBS and the remaining pellet was resuspended in sPBS.

\section{Cell counts}

The cell counts were assessed for both the oPMNs and cPMNs with a Muse Cell Analyzer (Merck Millipore, Darmstadt, Germany) using the Counts and Viability Assay kit (Merck Millipore, Darmstadt, Germany) according to the manufacturer's instructions.

\section{Cell cycle analysis}

The apoptosis levels of the oPMNs and cPMNs were measured by double staining with fluorescein isothiocyanate (FITC)-conjugated Annexin V and propidium iodide (PI) using the BD Pharmingen FITC-Annexin V Apoptosis Detection Kit (BD Biosciences, San Diego, CA, USA) according to the manufacturer's instructions. Briefly, $2.5 \mu \mathrm{L}$ FITC-Annexin V and $2.5 \mu \mathrm{L}$ PI were added to a $50-\mu \mathrm{L}$ cell suspension. After $15 \mathrm{~min}$ incubation in the dark, $200 \mu \mathrm{L}$ of Annexin V binding buffer $\left(0.01 \mathrm{~mol} \cdot \mathrm{L}^{-1} \mathrm{HEPES} / \mathrm{NaOH} \mathrm{pH} \quad 7.4,0.14 \mathrm{~mol} \cdot \mathrm{L}^{-1} \mathrm{NaCl}\right.$, $2.5 \mathrm{mmol} \cdot \mathrm{L}^{-1} \mathrm{CaCl}_{2}$ ) was added to the mixture. A flow cytometric 
analysis was performed within $1 \mathrm{~h}$ on a BD Accuri C6 flow cytometer (BD Biosciences, San Diego, CA, USA).

\section{Cellular activation assay}

The cell membrane expression of CD11b, CD63, and CD66b was measured. The samples were incubated for $30 \mathrm{~min}$ on ice with one of the following mixes: (A) mouse anti-human phycoerythrin (PE)conjugated $\mathrm{CD} 63+$ mouse anti-human FITC-conjugated CD66b, (B) mouse anti-human PE-conjugated CD11b+mouse anti-human FITCconjugated CD66b, or (C) IgG1-PE isotype control and IgG1-FITC isotype control (all from BD Biosciences, San Diego, CA, USA) at a final concentration of $10 \mu \mathrm{g} \cdot \mathrm{mL}^{-1}$. The PMNs were gated according to their relative size (forward scatter) and granularity (sideward scatter). The expression of the cell surface markers was calculated as the mean fluorescence intensity (MFI) and corrected for non-specific binding of isotype control antibodies.

\section{Bacterial culture}

Fusobacterium nucleatum strain ATCC10953 was commercially obtained (DSMZ, Braunschweig, Germany). F. nucleatum was grown anaerobically ( $80 \%$ nitrogen, $10 \%$ carbon dioxide, and $10 \%$ hydrogen) in a brain-heart infusion broth supplemented with $5 \mu \mathrm{g} \cdot \mathrm{mL}^{-1}$ hemin (Sigma-Aldrich Chemie B.V., Zwijndrecht, Netherlands) and $1 \mu \mathrm{g} \cdot \mathrm{mL}^{-1}$ menadione (Sigma-Aldrich Chemie B.V., Zwijndrecht, Netherlands). The bacteria were isolated from the broth cultures by centrifugation, washed twice in sterile PBS before dilution with sterile PBS, to yield a final suspension of $4 \times 10^{8}$ cells per $\mathrm{mL}$, which was stored at $-20^{\circ} \mathrm{C}$.

\section{ROS assay}

The cPMNs were diluted to a concentration of $1.0 \times 10^{6}$ cells per $\mathrm{mL}$. The chemiluminescence was measured using a Wallace Victor ${ }^{2} 1420$ (Perkin-Elmer, Boston, MA, USA). All the samples were measured in duplicate using opaque 96-microwell plates that had been preblocked at $4{ }^{\circ} \mathrm{C}$ overnight with PBS-containing $1.0 \%$ bovine serum albumin. Each microwell contained $6 \mathrm{U}$ horseradish peroxidase and either $3 \mathrm{mmol} \cdot \mathrm{L}^{-1}$ luminol (to detect total ROS production) or $3 \mathrm{mmol} \cdot \mathrm{L}^{-1}$ isoluminol (to detect extracellular ROS production). The peak of the ROS production in relative light units (RLUs) was recorded from unstimulated PMNs and from the stimulated PMNs being incubated with $30 \mu \mathrm{L} \mathrm{F}$. nucleatum for $30 \mathrm{~min}$ at $37^{\circ} \mathrm{C}$. The RLUs were corrected for the number of viable cells per well for both the cPMNs and oPMNs and expressed as peak ROS per viable cell.

\section{Statistical analysis}

The analyses were performed using SPSS Statistics 21.0 software (IBM, Chicago, IL, USA). The normality of the data was confirmed by the Kolomogorov-Smirnov test. The means and standard deviations were calculated. The comparisons between the oPMNs and cPMNs were analyzed by paired $t$-tests. In addition, a multivariate analysis of covariance (MANCOVA) was performed to control for potential confounders using donor gender as a fixed factor and participant age, mean plaque and BOMP scores as covariates. oPMNs and cPMNs parameters in males and females were compared using independent $t$-tests. Correlations between parameters were assessed by Pearson's correlation coefficient. To account for multiple testing and reduce the chances of obtaining false-positive results, a Bonferroni correction was applied by dividing the $\alpha$-value by the number of tested variables (plaque, BOMP, oPMN counts, cPMN counts, cell cycle analysis, cell activation analysis, and ROS production). ${ }^{30} P$-values $\leq 0.007(0.05 / 7)$ were considered statistically significant.

\section{RESULTS}

\section{Study population}

Before the start of the study, 336 potential participants were screened at the dental clinic in a separate session. ${ }^{24-25} \mathrm{~A}$ total of 47 subjects were excluded (23 male subjects and 24 female subjects) based on the inclusion and exclusion criteria. Also, 10 subjects took part in a pilot study and were not included in the final data, and 11 dropped out during the study because of scheduling problems. The final study population consisted of 268 healthy volunteers (56\% male participants), with a mean age of 23 years on the day of their appointment and an age range of 18-32 years (Table 1). The mean plaque score in terms of percentage was $33.3 \%$ and was higher in males compared to females (Table $1, P<0.001$ ). The total mean percentage BOMP score was $20.1 \%$, and was not significantly different relative to donor gender (Table 1).

\section{PMN counts}

The results of the oPMN and the cPMN counts are shown in Table 1. On average, a total of $1.1 \times 10^{6} \pm 0.9 \times 10^{6}$ oPMNs were collected per subject from four rinses. The cell counts varied among the individuals (range $0.07 \times 10^{6}-7.6 \times 10^{6}$ ) with comparable numbers isolated from males and females $(P=0.015)$. On average, $8.4 \times 10^{6} \pm 6.2 \times 10^{6}$ cPMNs (range $0.9 \times 10^{6}-82.0 \times 10^{6}$ ) were isolated from a $6 \mathrm{~mL}$ tube of blood, with a tendency for higher $\mathrm{CPMN}$ numbers from females $(P=0.008)$. No correlation was found between the number of the oPMNs and cPMNs $(r=-0.08, P=0.216)$.

Table 1 Characteristics of the study population stratified according to gender

\begin{tabular}{|c|c|c|c|c|c|}
\hline Parameters & Total $(n=268)$ & Males $(n=150)$ & Females $(n=118)$ & $P$-value* & Adjusted $P$-value ${ }^{\dagger}$ \\
\hline \multicolumn{6}{|c|}{ Dental characteristics (\%) } \\
\hline Plaque & $33.3 \pm 21.1(0.0-100.0)$ & $37.9 \pm 21.5(1.2-100.0)$ & $27.3 \pm 19.1(0.0-90.5)$ & $<0.001$ & \\
\hline BOMP & $20.1 \pm 12.4(1.2-64.3)$ & $21.2 \pm 13.3(1.2-64.3)$ & $18.6 \pm 11.1(1.2-52.4)$ & 0.081 & \\
\hline \multicolumn{6}{|c|}{ Counts $\left(\times 10^{6}\right)$} \\
\hline oPMNs ${ }^{\ddagger}$ & $1.1 \pm 0.9(0.07-7.7)$ & $1.2 \pm 1.0(0.07-7.7)$ & $1.0 \pm 0.7(0.09-4.1)$ & 0.015 & 0.231 \\
\hline $\mathrm{cPMNs}^{\S}$ & $8.4 \pm 6.2(0.9-82.0)$ & $7.5 \pm 6.7(0.9-82.0)$ & $9.5 \pm 5.3(1.1-32.8)$ & 0.008 & 0.042 \\
\hline
\end{tabular}

BOMP, bleeding on marginal probing; CPMN, circulatory polymorphonuclear neutrophils; oPMN, oral polymorphonuclear neutrophils.

Results for dental characteristics and counts of oPMNs and cPMNs. Values represent means \pm standard deviations (range).

*Independent $t$-tests, $P$-values $\leq 0.007$ were considered statistically significant and are shown in bold.

${ }^{\dagger}$ Multivariate analysis of covariance, using donor gender as a fixed factor and participant age, mean plaque and BOMP scores as covariates. Adjusted $P$-values $\leq 0.007$ were considered statistically significant.

¥Total oPMNs collected after $4 \times 30 \mathrm{~s}$ rinsing with 4.5-min intermission, after the isolation and purification steps.

§Total cPMNs obtained from a $6 \mathrm{~mL}$ tube of blood, after the isolation and purification steps. 


\section{Cell cycle analysis}

Results of the cell cycle analysis for the oPMNs and cPMNs are shown in Figure 1. The non-apoptotic cells with fully intact membranes are negative for both PI and FITC, and the cells that stain positive for Annexin V FITC but negative for PI are in an early stage of apoptosis, whereas the cells that stain positive for both Annexin V and PI are either in a later stage of apoptosis or have completely lost cellular integrity. ${ }^{31}$ An appreciable proportion of the oPMNs $(22.6 \% \pm 14.0 \%)$ was found to be non-apoptotic, but this subset was lower compared with the cPMNs $(41.3 \% \pm 22.3 \%, P<0.001$, Figure 1a). Furthermore, fewer oPMNs $(22.9 \% \pm 5.6 \%)$ than cPMNs $(49.7 \% \pm 19.7 \%, P<0.001$, Figure 1b) were found in an early stage of apoptosis, whereas a higher proportion of the oPMNs $(53.1 \% \pm 12.9 \%)$ was found in a later stage of apoptosis/necrosis compared to cPMNs $(8.5 \% \pm 6.2 \%, P<0.001$,
Figure 1c). No differences in relation to donor gender were found (Table 2).

\section{Cellular activation}

The expressions of the three PMN activation markers are shown in Figure 2. All three markers were expressed higher on the oPMNs compared with the cPMNs (CD11b $12.8 \times 10^{4} \pm 6.8 \times 10^{4}$ versus $3.8 \times 10^{4} \pm 2.2 \times 10^{4}$ respectively, CD63 $10.1 \times 10^{4} \pm 5.9 \times 10^{4}$ versus $1.1 \times 10^{4} \pm 1.7 \times 10^{4}$ respectively, CD66b $4.9 \times 10^{4} \pm 4.9 \times 10^{4}$ versus $1.2 \times 10^{4} \pm 1.8 \times 10^{4}$ respectively, $P<0.001$ for all three markers). The cellular expression of CD63 was positively correlated between the oPMNs and cPMNs $\left(r_{\mathrm{CD} 63}=0.38, P<0.001\right)$. No correlations were observed for $\mathrm{CD} 11 \mathrm{~b}$ and $\mathrm{CD} 66 \mathrm{~b}$ between the oPMNs and cPMNs $\left(r_{\mathrm{CD} 11 \mathrm{~b}}=0.14, P=0.026\right.$, and $r_{\mathrm{CD} 66 \mathrm{~b}}=0.12$,
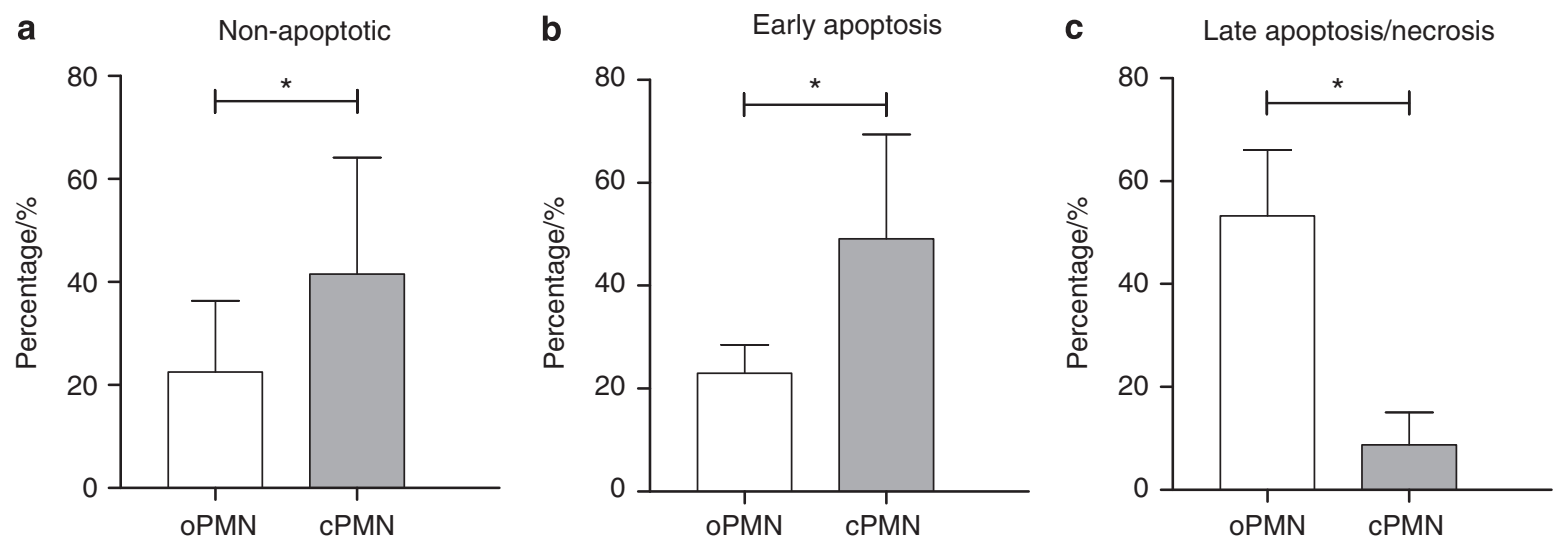

Figure 1 Cell cycle staining. (a) Annexin $\mathrm{V}^{-} / \mathrm{PI}^{-}$cells were considered non-apoptotic. (b) Annexin $\mathrm{V}^{+} / \mathrm{PI}^{-}$cells were considered in an early stage of apoptosis. (c) Annexin $\mathrm{V}^{+} / \mathrm{PI}^{+}$were in a late stage of apoptosis/necrosis. Data are mean percentages \pm standard deviation. ${ }^{*}$ Comparison oPMNs and $\mathrm{cPMNs}, \mathrm{P}<0.001$. cPMN, circulatory polymorphonuclear neutrophils; oPMN, oral polymorphonuclear neutrophils; PI, propidium iodide.

Table 2 Results for cell cycle analysis, cell activation status and ROS production for oPMNs and cPMNs, stratified according to gender

\begin{tabular}{|c|c|c|c|c|c|c|c|c|}
\hline \multirow[b]{2}{*}{ Parameters } & \multicolumn{4}{|c|}{ oPMNs } & \multicolumn{4}{|c|}{ cPMNs } \\
\hline & Males $(n=150)$ & Females $(n=118)$ & $P$-value* & Adjusted $P$-value ${ }^{\dagger}$ & Males $(n=150)$ & Females $(n=118)$ & $P$-value* & Adjusted $P$-value ${ }^{\dagger}$ \\
\hline \multicolumn{9}{|c|}{ Cell cycle analysis (\%) } \\
\hline Non-apoptotic & $22.6 \pm 13.9$ & $22.6 \pm 14.2$ & 0.996 & 0.582 & $38.7 \pm 21.6$ & $44.7 \pm 22.8$ & 0.028 & 0.005 \\
\hline Early apoptosis & $22.9 \pm 5.8$ & $23.1 \pm 5.4$ & 0.800 & 0.365 & $52.0 \pm 19.4$ & $46.7 \pm 19.8$ & 0.030 & 0.026 \\
\hline Late apoptosis & $53.1 \pm 12.8$ & $53.2 \pm 13.0$ & 0.922 & 0.459 & $8.6 \pm 5.9$ & $8.3 \pm 6.5$ & 0.700 & 0.014 \\
\hline \multicolumn{9}{|c|}{ Cell activation $\left(\mathrm{MFI} \times 10^{4}\right)$} \\
\hline CD11b & $12.4 \pm 7.1$ & $13.2 \pm 6.5$ & 0.337 & 0.301 & $3.7 \pm 2.2$ & $3.8 \pm 2.2$ & 0.702 & 0.375 \\
\hline CD63 & $9.7 \pm 5.6$ & $10.6 \pm 6.2$ & 0.210 & 0.851 & $0.9 \pm 1.6$ & $1.4 \pm 1.7$ & 0.012 & 0.686 \\
\hline CD66b & $4.5 \pm 5.2$ & $5.6 \pm 4.5$ & 0.049 & 0.043 & $1.0 \pm 1.8$ & $1.3 \pm 1.9$ & 0.239 & 0.555 \\
\hline \multicolumn{9}{|c|}{ Total ROS (RLU × $\left.10^{-3}\right)$} \\
\hline Unstimulated & $5.3 \pm 10.5$ & $3.7 \pm 3.9$ & 0.133 & 0.098 & $0.9 \pm 0.2$ & $1.1 \pm 0.2$ & 0.001 & 0.007 \\
\hline F. nucleatum & $18.5 \pm 46.7$ & $12.3 \pm 17.3$ & 0.168 & 0.218 & $10.9 \pm 7.1$ & $11.3 \pm 8.7$ & 0.649 & 0.925 \\
\hline \multicolumn{9}{|c|}{ Extracellular ROS $\left(\mathrm{RLU} \times 10^{-3}\right)$} \\
\hline Unstimulated & $3.4 \pm 4.0$ & $3.5 \pm 3.8$ & 0.754 & 0.489 & $1.0 \pm 0.2$ & $1.1 \pm 0.2$ & $<0.001$ & 0.007 \\
\hline F. nucleatum & $5.7 \pm 9.7$ & $5.0 \pm 5.9$ & 0.454 & 0.250 & $2.6 \pm 1.1$ & $2.7 \pm 1.4$ & 0.554 & 0.532 \\
\hline
\end{tabular}

cPMN, circulatory polymorphonuclear neutrophils; MFI, mean fluorescence intensity; oPMN, oral polymorphonuclear neutrophils; RLU, relative light units; ROS, reactive oxygen species.

Values represent means \pm standard deviations.

*Independent $t$-tests, $P$-values $\leq 0.007$ were considered statistically significant and are shown in bold.

${ }^{\dagger}$ Multivariate analysis of covariance, using donor gender as a fixed factor and participant age, mean plaque and BOMP scores as covariates. Adjusted $P$-values $\leq 0.007$

were considered statistically significant and are shown in bold. 
$P=0.050)$. Also, no differences were found relative to donor gender (Table 2).

\section{ROS}

The levels of ROS produced by the oPMNs and the cPMNs are presented in Figure 3; Figure 3a shows the total ROS production (luminol dependent). The levels of total constitutive ROS from the oPMNs were higher than from the cPMNs $\left(4.6 \times 10^{-3} \pm 8.3 \times 10^{-3}\right.$ RLU versus $1.0 \times 10^{-3} \pm 0.2 \times 10^{-3}$ RLU respectively, $P<0.001$ ). In response to F. nucleatum stimulation, the levels of the total ROS increased 3.4-fold on average for the oPMNs compared with the unstimulated ROS $\left(15.8 \times 10^{-3} \pm 36.8 \times 10^{-3}\right.$ RLU, $\left.P<0.001\right)$. Under the same conditions, the cPMNs upregulated the total ROS production, on average 10.8-fold, compared with the unstimulated ROS $\left(11.1 \times 10^{-3} \pm 7.8 \times 10^{-3}\right.$ RLU, $\left.P<0.001\right)$. The total ROS production in response to $F$. nucleatum was not significantly different between the oPMNs and cPMNs $(P=0.042)$.

A similar pattern of response was observed when the extracellular ROS levels (isoluminol dependent) were measured (Figure 3b). The extracellular constitutive ROS was higher for the oPMNs than for the cPMNs $\left(3.4 \times 10^{-3} \pm 3.9 \times 10^{-3}\right.$ RLU versus $1.1 \times 10^{-3} \pm 0.2 \times 10^{-3}$ RLU, respectively, $P<0.001)$. Following $F$. nucleatum stimulation, the extracellular ROS increased 1.6-fold for the oPMNs $\left(5.4 \times 10^{-3} \pm 8.2 \times 10^{-3} \mathrm{RLU}, P<0.001\right)$ and 2.5 fold for the cPMNs $\left(2.6 \times 10^{-3} \pm 1.2 \times 10^{-3} \mathrm{RLU}, P<0.001\right)$. The extracellular ROS released in response to $F$. nucleatum was higher for the oPMNs compared with the cPMNs $(P<0.001)$.

In the absence of stimulation, the luminescence values in the luminol samples were not significantly different from those in the isoluminol samples. This suggests that the constitutive ROS measured was mainly extracellular for both the oPMNs and the cPMNs (Figure $3 \mathrm{a}$ and $3 \mathrm{~b}$ ). The levels of constitutive ROS on the oPMNs were positively correlated to the ROS production in response to the bacterial stimulus ( $r_{\text {total }}$ ROS $=0.90, \quad r_{\text {extracellular }} \operatorname{ROS}=0.79$, $P<0.001$ ). This was not the case for the cPMNs (cPMNs: $r_{\text {total }}$ ROS $=-0.07, P=0.262, r_{\text {extracellular ROS }}=-0.04, P=0.565$ ). In addition, cPMNs from females expressed higher levels of constitutive ROS production compared with males (Table 2, $P_{\text {total }}$ ROS $=0.001$, $\left.P_{\text {extracellular ROS }}<0.001\right)$.
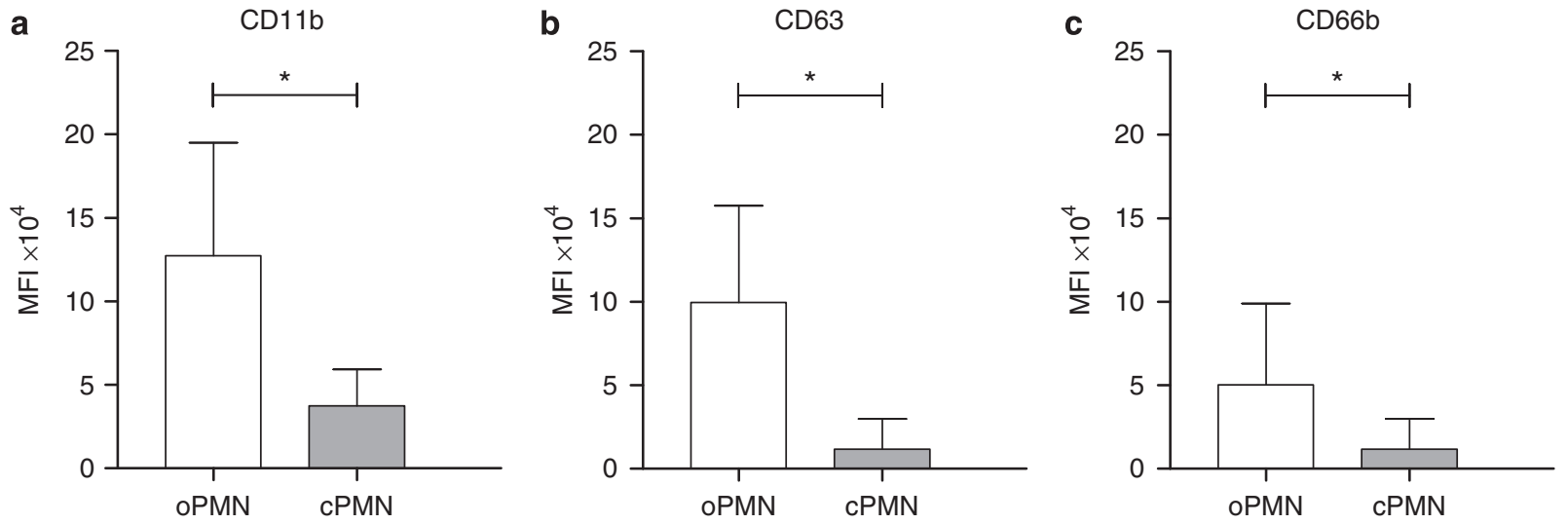

Figure 2 Cell surface markers were measured and corrected for non-specific binding of isotype control antibodies, indicating cell activation. (a) CD11b; (b) CD63; (c) CD66b. Data are means of the mean fluorescence intensity \pm standard deviation, *comparison oPMNs and cPMNs, $P<0.001$. cPMN, circulatory polymorphonuclear neutrophils; MFI, mean fluorescence intensity; oPMN, oral polymorphonuclear neutrophils.
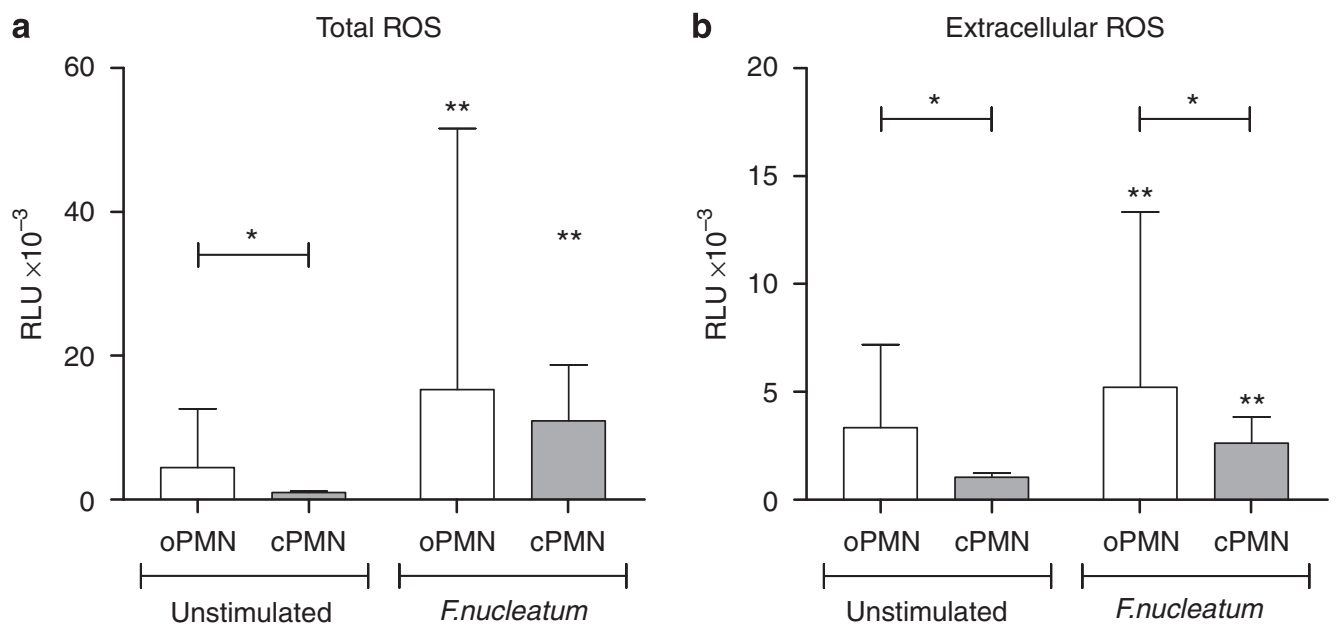

Figure 3 Total ROS production and extracellular ROS production in unstimulated samples and samples stimulated with $F$. nucleatum, assessed using luminol and isoluminol, respectively. (a) Total ROS; (b) extracellular ROS. Maximal RLUs were recorded and corrected for the number of viable cells. Data are means RLUs \pm standard deviation, *comparison oPMNs and cPMNs, $P<0.001$; ${ }^{* *}$ comparison unstimulated and $F$. nucleatum, $P<0.001 . c P M N$, circulatory polymorphonuclear neutrophils; oPMN, oral polymorphonuclear neutrophils; RLUs, relative light units; ROS, reactive oxygen species. 
Table 3 Correlations between BOMP and oPMN parameters stratified according to gender

BOMP

\begin{tabular}{|c|c|c|c|c|c|c|}
\hline oPMN parameters & $r$ & $P$-value* & $r$ & $P$-value* & $r$ & $P$-value* \\
\hline \multicolumn{7}{|l|}{ Cell cycle analysis } \\
\hline Non-apoptotic & -0.018 & 0.768 & 0.002 & 0.979 & -0.049 & 0.599 \\
\hline Early apoptosis & 0.007 & 0.909 & -0.027 & 0.743 & 0.067 & 0.470 \\
\hline \multicolumn{7}{|l|}{ Cell activation } \\
\hline $\mathrm{CD} 11 \mathrm{~b}$ & 0.117 & 0.056 & 0.110 & 0.181 & 0.149 & 0.107 \\
\hline CD63 & 0.003 & 0.963 & -0.012 & 0.886 & 0.043 & 0.641 \\
\hline CD66b & 0.015 & 0.811 & 0.047 & 0.567 & -0.007 & 0.943 \\
\hline \multicolumn{7}{|l|}{ Total ROS } \\
\hline Unstimulated & 0.142 & 0.020 & 0.179 & 0.028 & -0.018 & 0.845 \\
\hline
\end{tabular}

BOMP, bleeding on marginal probing; oPMN, oral polymorphonuclear neutrophils; ROS, reactive oxygen species.

*Pearson's correlations, $P$-values $\leq 0.007$ were considered statistically significant.

\section{Exploratory analysis}

Mean percentage BOMP scores was positively correlated at an individual level with the percentage plaque score $(r=0.292$, $P<0.001)$, both for males $(r=0.261, P=0.001)$, and females $(r=0.301, P=0.001)$. Furthermore, mean \% BOMP scores tended to correlate with oPMN counts $(r=0.163, P=0.008$, Table 3$)$; this tendency was observed only in males. The MANCOVAgenerated adjusted $P$-values are presented in Tables 1 and 2 (adjusted $P \leq 0.007$ was considered statistically significant). All the significant differences generated by the $t$-tests were confirmed in the MANCOVA model. Furthermore, females showed a higher percentage of nonapoptotic cPMNs compared to males (Table 2, $44.7 \pm 22.8,38.7 \pm 21.6$, respectively adjusted $P=0.005)$.

\section{DISCUSSION}

The goal of the present study was to describe the numbers and characteristics of PMNs isolated from the oral cavity in a large sample of healthy young individuals. The rationale was to obtain possible reference values for the oPMN characteristics associated with a state of homeostasis. The oPMNs were compared with the cPMNs using flow cytometric analysis of the PMN activation state and chemiluminescence analysis of functional ROS production capabilities. The main finding is that the viable oPMNs obtained from oral rinses were capable of performing innate immune responses comparable to the cPMNs. This result indicates a functional role for oPMNs in oral health maintenance.

The numbers of oPMNs available after isolation together with the constraints for timely processing fresh cells for this large group were limiting our choice for the functional assays. We could not assess phagocytosis and NETosis. However, the complete set of measurements (ROS production and flow cytometric evaluation of cell cycle and activation status) offered the possibility to gain valuable insights into oPMNs as potential functional contributors in the oral cavity.

In this study, a tendency for higher cPMN numbers were found in female compared with male donors, with significantly higher constitutive ROS levels. These findings confirms observations from earlier studies that reported up to $20 \%$ higher numbers of circulating PMNs showing increased ROS levels in females. ${ }^{32-33}$ These authors suggested that the increased PMN survival, that is, delayed apoptosis, and observed ROS levels may be influenced by hormones such as estradiol and progesterone. Interestingly, the oPMN numbers were comparable relative to donor gender. Also, no correlation was found between the numbers of the oPMNs and the cPMNs. Although the cPMNs reflect the general inflammatory status of an individual, the oPMN counts vary with local inflammatory conditions in the oral cavity. Early studies revealed higher rates of PMN migration into the oral cavity in the presence of gingival inflammation, ${ }^{34}$ and the lowest oPMN numbers have been reported after tooth extractions. ${ }^{35-36}$ Local inflammatory changes were also related to oPMNs in our study group, since the oPMN counts tended to correlate with BOMP.

Analysis of the PMN life cycle revealed that (i) the proportion of viable oPMNs was significantly lower compared with cPMNs and (ii) the cell cycle stage of oPMNs was skewed towards later stages of apoptosis/necrosis. The longevity of PMNs can increase several-fold upon activation, which ensures the presence of primed functional PMNs at a site of inflammation. ${ }^{37}$ Also, bacterially derived endotoxin present in the oral cavity might have an inhibitory effect on apoptosis and, thus, PMNs in the oral cavity most likely die through necrosis, rather than through a programmed cell death. ${ }^{20}$

Upon phagocytosis, PMNs become activated, and degranulation occurs as well as intracellular and extracellular ROS production. This study shows that ex vivo unstimulated oPMNs were 3-9 times more activated, as seen by their granular content release, and showed higher baseline ROS levels compared with the cPMNs. Similar findings have been reported before; significantly higher levels of $\mathrm{H}_{2} \mathrm{O}_{2}$ were observed from the resting oPMNs than from cPMNs. ${ }^{19}$ We suggest that the increased cellular activation, that is, degranulation, and level of constitutive ROS production, may be due to priming of PMNs after exposure to microorganisms in the oral biofilm.

Furthermore, the oPMNs from healthy subjects were able to mount an oxidative burst showing increased ROS levels compared to baseline, after bacterial stimulation with non-opsonized F. nucleatum. These 
levels of ROS were comparable to the levels produced by the cPMNs, which indicates an effective innate immune response as well as their potential for phagocytosis. This result corroborates previous reports showing unimpaired levels of ROS generated by oPMNs from healthy donors in response to fMLP, a bacterium-derived stimulus. ${ }^{19,38}$ In contrast, two other studies found that oPMNs isolated from whole saliva retained some functional activity, however it was lower than that of the cPMNs, prompting the authors to regard salivary PMNs as being functionally impaired. ${ }^{39-40} \mathrm{~A}$ reason for the apparently contrasting results may be the different collection methods. In the latter two studies, whole saliva was collected as a source for the oPMNs. ${ }^{39-40}$ Of note is that the integrity of PMNs is preserved in the mucus phase of oral secretions, ${ }^{41}$ whereas saliva has been shown to inhibit PMN functions, such as phagocytosis, ROS production and killing bacteria. ${ }^{42}$ The inhibitory effects of saliva on PMN functions have been attributed to salivary hypotonicity and $\mathrm{pH}$. To avoid these possible effects, we limited the contact of the oPMNs with saliva in the current study by using serial rinses with isotonic buffer.

During PMN activation, primary and secondary granules are mobilized and fuse with the plasma membrane. The degranulation of primary granules was analyzed by the expression of CD63, and the secondary granules were analyzed by measuring CD66b. Furthermore, $\mathrm{CD} 11 \mathrm{~b}$ expression was assessed because of its involvement in PMN adhesion and transepithelial migration. ${ }^{43}$ Interestingly, the expression of CD63 on the oPMNs was positively correlated with CD63 expression on the cPMNs; and a similar trend was found CD66b and CD11b. This suggests that primed PMNs found in the systemic circulation maintain the same trait of activation after transmigration into the oral cavity.

In recent years, the quantification of oPMNs has been proposed as a screening tool for various conditions, ranging from gingivitis and periodontitis to systemic inflammation after cardiac bypass or bone marrow transplantation involving extensive neutropenia. ${ }^{12,16-17,44-46}$ This study analyzed oPMNs associated with the normal physiological state of the oral cavity, that is, non-diseased oral cavity, and compared their characteristics with the cPMNs. We were able to isolate viable oPMNs from all the participants within this sizeable population of systemically and orally healthy young individuals, thus our results can become future reference values in the research for pathological changes associated with oral or systemic conditions.

Unresolved issues remain to fully comprehend the specific role and functional activities of PMNs in oral health maintenance, such as phagocytosis capabilities, cell migration, and the effects of aging. ${ }^{47-48}$ On the basis of our findings, we confirm that oPMNs from males and females have a comparable physiologic role and that evaluating the oPMNs could be useful in assessing the level of health in the oral ecosystem, ${ }^{47}$ in a similar way that $\mathrm{CPMNs}$ are used for testing for suspected infections or other conditions. The concept of counting the oPMNs using rinse samples has been proposed before as a possible screening method to indicate the oral inflammatory load. ${ }^{45}$ Further development of this approach may enable dental practitioners to measure the inflammatory load using a chairside method as opposed to relying on clinical parameters alone.

In conclusion, we observed oPMNs in a later stage of their life cycle compared with cPMNs, and found them still capable of performing innate immune functions, which is highly suggestive of their contribution in oral health maintenance.

\section{ACKNOWLEDGEMENTS}

We thank Eva Wartewig, Shevan Abdokah, Wijnand Teeuw, and Elena Alvarez for their contributions to sample collection. We also thank Nienke Hennequin-
Hoenderdos, Dagmar Slot, and Eveline van der Sluijs for their contribution in developing the clinical protocol and for coordinating and monitoring the clinical part of this study. The Departments of Periodontology and Preventive Dentistry are supported by a grant from the University of Amsterdam for research into the focal point "Oral Infections and Inflammation". The study was financed in part by ACTA Dental Research BV (ADR). ADR has received funding from TI Food and Nutrition, a public-private partnership on precompetitive research in food and nutrition.

1 Keijser BJ, Zaura E, Huse SM et al. Pyrosequencing analysis of the oral microflora of healthy adults. J Dent Res 2008; 87(11): 1016-1020.

2 Wade WG. The oral microbiome in health and disease. Pharmacol Res 2013; 69(1): 137-143.

3 Szpaderska AM, Zuckerman JD, DiPietro LA. Differential injury responses in oral mucosal and cutaneous wounds. J Dent Res 2003; 82(8): 621-626.

4 Dale BA, Fredericks LP. Antimicrobial peptides in the oral environment: expression and function in health and disease. Curr Issues Mol Biol 2005; 7(2): 119-133.

5 Sansonetti PJ. To be or not to be a pathogen: that is the mucosally relevant question. Mucosal Immunol 2011; 4(1): 8-14.

6 Fournier BM, Parkos CA. The role of neutrophils during intestinal inflammation. Mucosal Immunol 2012; 5(4): 354-366.

7 Cross AR, Segal AW. The NADPH oxidase of professional phagocytes-prototype of the NOX electron transport chain systems. Biochim Biophys Acta 2004; 1657(1): 1-22.

8 Chapple IL, Matthews JB. The role of reactive oxygen and antioxidant species in periodontal tissue destruction. Periodontol 2000 2007; 43: 160-232.

9 Chapple IL. Reactive oxygen species and antioxidants in inflammatory diseases. J Clin Periodontol 1997; 24(5): 287-296.

10 Kobayashi SD, DeLeo FR. Role of neutrophils in innate immunity: a systems biologylevel approach. Wiley Interdiscip Rev Syst Biol Med 2009; 1(3): 309-333.

11 Aboodi GM, Goldberg MB, Glogauer M. Refractory periodontitis population characterized by a hyperactive oral neutrophil phenotype. J Periodontol 2011; 82(5): 726-733.

12 Aps JK, Van den Maagdenberg K, Delanghe JR et al. Flow cytometry as a new method to quantify the cellular content of human saliva and its relation to gingivitis. Clin Chim Acta 2002; 321(1/2): 35-41.

13 Bender JS, Thang H, Glogauer M. Novel rinse assay for the quantification of oral neutrophils and the monitoring of chronic periodontal disease. J Periodont Res 2006; 41(3): 214-220.

14 Bhadbhade SJ, Acharya AB, Thakur S. Correlation between probing pocket depth and neutrophil counts in dental plaque, saliva, and gingival crevicular fluid. Quintessence Int 2012; 43(2): 111-117.

15 Lakschevitz FS, Aboodi GM, Glogauer M. Oral neutrophil transcriptome changes result in a pro-survival phenotype in periodontal diseases. PLoS One 2013; 8(7): e68983.

16 Moosani A, Sigal MJ, Glogauer M et al. Evaluation of periodontal disease and oral inflammatory load in adults with special needs using oral neutrophil quantification. Spec Care Dentist 2014; 34(6): 303-312.

17 Wilcox ME, Charbonney E, d'Empaire PP et al. Oral neutrophils are an independent marker of the systemic inflammatory response after cardiac bypass. J Inflamm (Lond) 2014; 11(1): 32

18 Fredriksson MI. Effect of priming in subpopulations of peripheral neutrophils from patients with chronic periodontitis. J Periodontol 2012; 83(9): 1192-1199.

19 Ashkenazi M, Dennison DK. A new method for isolation of salivary neutrophils and determination of their functional activity. J Dent Res 1989; 68(8): 1256-1261.

20 Crawford JM, Wilton JM, Richardson P. Neutrophils die in the gingival crevice, periodontal pocket, and oral cavity by necrosis and not apoptosis. J Periodontol 2000; 71(7): 1121-1129.

21 Lakschevitz FS, Aboodi GM, Glogauer M. Oral neutrophils display a site-specific phenotype characterized by expression of T-cell receptors. J Periodontol 2013; 84(10): 1493-1503.

22 Slebioda Z, Szponar E, Kowalska A. Defensins and their role in the maintenance of the oral cavity homeostasis - a literature review. Cent Eur J Immunol 2013; 38(1): 111-117.

23 Lakschevitz FS, Visser MB, Sun C et al. Neutrophil transcriptional profile changes during transit from bone marrow to sites of inflammation. Cell Mol Immunol 2015; 12(1): 53-65.

24 Oliveira SC, Slot DE, Celeste RK et al. Correlations between two different methods to score bleeding and the relationship with plaque in systemically healthy young adults. $J$ Clin Periodontol 2015; 42(10): 908-913.

25 Prodan A, Brand HS, Ligtenberg AJ et al. Interindividual variation, correlations, and sexrelated differences in the salivary biochemistry of young healthy adults. Eur J Oral Sci 2015; 123(3): 149-157.

26 Van Leeuwen M, Rosema N, Versteeg PA et al. Effectiveness of various interventions on maintenance of gingival health during 1 year-a randomized clinical trial. Int J Dent Hyg 2016; doi:10.1111/idh.12213. [Epub ahead of print].

27 al-Essa L, Niwa M, Kohno $\mathrm{K}$ et al. A proposal for purification of salivary polymorphonuclear leukocytes by combination of nylon mesh filtration and density-gradient method: a validation by superoxide- and cyclic AMP-generating responses. Life Sci 1994: 55(17): PL333-PL338.

28 Van der Weijden GA, Timmerman MF, Nijboer A et al. A comparative study of electric toothbrushes for the effectiveness of plaque removal in relation to toothbrushing duration. Timerstudy. J Clin Periodontol 1993; 20(7): 476-481. 
29 Lie MA, Timmerman MF, van der Velden $U$ et al. Evaluation of 2 methods to assess gingival bleeding in smokers and non-smokers in natural and experimental gingivitis. J Clin Periodontol 1998; 25(9): 695-700.

30 Ranstam J. Multiple p-values and Bonferroni correction. Osteoarthritis Cartilage 2016; 24(5): 763-764.

31 Majewska E, Sulowska Z, Baj Z. Spontaneous apoptosis of neutrophils in whole blood and its relation to apoptosis gene proteins. Scand J Immunol 2000; 52(5): 496-501.

32 Bain BJ, England JM. Normal haematological values: sex difference in neutrophil count. Br Med J 1975; 1(5953): 306-309.

33 Molloy EJ, O'Neill AJ, Grantham JJ et al. Sex-specific alterations in neutrophil apoptosis: the role of estradiol and progesterone. Blood 2003; 102(7): 26532659.

34 Calonius PE. The leukocyte count in saliva. Oral Surg Oral Med Oral Pathol 1958; 11(1): 43-46.

35 Lantzman E, Michman J. Leukocyte counts in the saliva of adults before and after extraction of teeth. Oral Surg Oral Med Oral Pathol 1970; 30(6): 766-773.

36 Wright DE. Leucocytes in the saliva of edentulous and caries-free subjects. Arch Oral Biol 1962; 7: 581-585.

37 Kolaczkowska E, Kubes P. Neutrophil recruitment and function in health and inflammation. Nat Rev Immunol 2013; 13(3): 159-175.

38 Ueta E, Osaki T, Yoneda $\mathrm{K}$ et al. Functions of salivary polymorphonuclear leukocytes (SPMNs) and peripheral blood polymorphonuclear leukocytes (PPMNs) from healthy individuals and oral cancer patients. Clin Immunol Immunopathol 1993; 66(3): 272-278.

39 Lukac J, Mravak-Stipetić M, Knezević M et al. Phagocytic functions of salivary neutrophils in oral mucous membrane diseases. J Oral Pathol Med 2003; 32(5): 271-274.

40 Scully C. Phagocytic and killing activity of human blood, gingival crevicular, and salivary polymorphonuclear leukocytes for oral streptococci. J Dent Res 1982; 61(5): 636-639.
41 Raeste AM. Degeneration of oral leukocytes. Scand J Dent Res 1972; 80(4): 285-291.

42 Saito K, Kato C, Teshigawara H. Saliva inhibits the chemiluminescence response, phagocytosis, and killing of Staphylococcus epidermidis by polymorphonuclear leukocytes. Infect Immun 1988; 56(8): 2125-2132.

43 Zemans RL, Colgan SP, Downey GP. Transepithelial migration of neutrophils: mechanisms and implications for acute lung injury. Am J Respir Cell Mol Biol 2009; 40(5): 519-535.

44 Forster C, Aboodi G, Lipton J et al. A non-invasive oral rinse assay predicts bone marrow engraftment and 6 months prognosis following allogeneic hematopoietic stem cell transplantation. J Oral Pathol Med 2012; 41(2): 165-170.

45 Landzberg M, Doering $\mathrm{H}$, Aboodi GM et al. Quantifying oral inflammatory load: oral neutrophil counts in periodontal health and disease. J Periodont Res 2015; 50(3): 330-336.

46 Petti S, Polimeni A, Berloco PB et al. Orofacial diseases in solid organ and hematopoietic stem cell transplant recipients. Oral Dis 2013; 19(1):18-36.

47 Hirschfeld J. Dynamic interactions of neutrophils and biofilms. J Oral Microbiol 2014; 6: 26102.

48 Wessels I, Jansen J, Rink L et al. Immunosenescence of polymorphonuclear neutrophils. ScientificWorldJournal 2010; 10: 145-160.

(c) (i) (2) This work is licensed under a Creative Commons AttributionCY NC SA NonCommercial-ShareAlike 4.0 International License. The images or other third party material in this article are included in the article's Creative Commons license, unless indicated otherwise in the credit line; if the material is not included under the Creative Commons license, users will need to obtain permission from the license holder to reproduce the material. To view a copy of this license, visit http:// creativecommons.org/licenses/by-nc-sa/4.0/ 